\title{
Neuigkeiten für die klinische Praxis
}

Gynäkologie

Thermographie in der Mamma-diagnostik

Mehr und mehr findet die Thermographie Eingang in die Mammadiagnostik der Arzt-praxis.

Deshalb bietet die Deutsche Gesell-schaft für Thermologie alien Anwendern und Interessenten regelmäßig Fortbildungs-kurse zur Einführung in die thermologische Untersuchung an. Anders als bei Mammo-graphie, Ultraschall und Palpation, die auf strukturelle, also morphologische Verände-rungen des Gewebes reagieren, zeigt die Thermographie funktionelle Störungen der Mamma an, bei denen sich die normale Symmetrie der Oberflächentemperaturver-teilung verändert. Deshalb kann die Thermographie auch nicht in Konkurrenz zu die-sen Methoden gesehen werden, sondern er-gänzt sie komplementär. Tumoren, aber auch Entzündungen führen durch Freiset-zung von Mediatoren zu erhöhtem Wärme-transport und erhöhter Wärme und damit zu auffälligen Thermogrammen. Eine Klassifi-kation in Th I-V (analog Papanicolaou) gibt gute Hinweise zur Differential diagnostik. Eine endgültige Diagnose läßt sich mit der Thermographie zwar nicht stellen, jedoch ist ein auffälliges Thermogramm stets ein Alarmzeichen, das der weiteren Abklärung bedarf. Besondere Vorteile der Thermographie liegen darin, daß es sich dabei um eine nicht-invasive, völlig risikolose Methode handelt, die beliebig oft angewandt werden kann. Funktionelle gehen zudem immer den strukturellen Veränderungen voraus. Daher gibt die Thermographie häufig schon Jahre vor einem mammographischen Befund erste Hinweise auf ein malignes Geschehen. Vor-aussetzung ist allerdings eine entsprechende »biologische Aktivität« und Malignität des Tumors. Sehr langsam wachsende Karzino-me geringerer Aggressivität werden von der Thermographie weniger häufig erfaßt. Ein-deutige Vorteile zeigt die Thermographie bei der Kontrolle von Frauen unter 35 Jah-ren, wo wegen der Dichte der Brüste Mam-mographie und Palpation oft genug keine zuverlässige Aussage erlauben, bei der Überwachung von Mastopathien und bei der Rezidivsuche im Narbenbereich nach Ablatio. Bisher viel zu wenig Beachtung findet bislang die Tatsache, daß sich bei einem Thermogramm der Klassifikation ThIII (unklar, kontrollbedürftig) bei negativem Befund von Palpation und Mammographie in annähernd 40\% innerhalb von 5 Jahren ein Karzinom nachweisen läßt. Damit gibt die Thermographie häufig das erste Alarm-

zeichen. Ein The-III-Thermogramm ist als Risikofaktor besonders aussagekräftig. Die preiswerte, risikolose und auch in der Arzt-praxis einfach anzuwendende Plattenther-mographie kann also bei routinemäßiger Anwendung die Früherkennung des Mam-makarzinoms wesentlich verbessern.

Auszeichnungen

Anläßlich der Jahrestagung der Deutschen Gesellschaft für Hämatologie und Onkologie vom 9. bis 12. Oktober 1983 in Münster, wurden der Artur-Pappenheim-Preis für Hämatologie und der Vincenz-Czerny-Preis für Onkologie verliehen. Der Preisträger des Artur-Pappenheim-Preises für Hämatologie 1983 ist Herr Dr. med. Axel A. Fauser, Freiburg, mit der Arbeit »Nachweis pluripotenter Stammzellpo-pulation aus dem menschlichen Knochen-mark mit myelolymphopoetischem Diffe-renzierungsprogramm«. Der Vincenz-Czerny-Preis für Onkologie 1983 
wurde Herrn Priv.-Doz. Dr. med. vet. Erich Eigenbrodt, Giessen, für die Arbeit »Zur Bedeutung der Pyruvatkinase-Isoen-zyme für die Steuerung des Kohlenhydrat-und Nucleinsäurestoffwechsels« verliehen. Anläßlich der Jahrestagung der Österreichi-schen Gesellschaft für Hämatologie und Onkologie in Klagenfurt vom 16. bis 17. September 1983, wurden der Wolfgang Denk-Preis und der Wilhelm-Türk-Preis durch den Präsidenten der Gesellschaft, Herrn Prof. Dr. K. Wagner, verliehen. Den Wolfgang-Denk-Preis erhielt P. Aiginger für seine Arbeit »Neue Aspekte in der Klinik und Fort-schritte in der Chemotherapie der malignen Hodentumoren«. Den Wilhelm-Türk-Preis erhielt Dr. G. Gastl zu gleichen Teilen mit Dr. D. Niederwieser und Dr. Ch. Huber für die Arbeit »Große, granuläre Lympho-zyten (GGL), eine neue Population mono-nukleärer Blutzellen«.

Radiologie

Neuer Angiographie-Arbeitsplatz für Indirekttechnik mit vollautomatischer 100-mm-BlattfilmKamera

Der angiographische Arbeitsplatz Compatix

A (Siemens) ist ein Röntgengerät für allge-

meine angiographische Untersuchungen

und Notfall-Angiographien mit Ausnahme

von Kardangio- und Zerebralangiographie.

Indirekt-Aufnahmetechnik ermöglicht

Durchleuchtungs-gezielte Aufnahmen, er-

leichtert die Handhabung und halt die Be-triebskosten niedrig. Compatix A besteht aus einem Untersuchungstisch mit quer ver-schiebbarer Lagerungsplatte und einem parallel zur TischLängsachse verfahrbaren Röntgenstrahler/Bildverstärker-System mit Fernseh- und 100-mmBlattfilm-Kamera. Die Anordnung Obertisch-Strahler/Unter-tisch-Bildverstärker ermöglicht guten Zu-gang zum Patienten. Der große Fokus-Ob-jekt-Abstand bietet - bei gleichzeitig geringem Objekt-Bildverstärker-Abstand - gün-stige Voraussetzungen für eine gute Aufnahmequalität. Strahlerstativ und bildgebendes System sind fest miteinander verbunden. Der Aufbau des Compatix A wird hierdurch sehr kompakt, außerdem wird die Montage wesentlich vereinfacht. Bei der peripheren Angiographie fährt das Stativ mit Strahler und Bildverstärker am ruhenden Patienten entlang. Eine unerwünschte Veränderung der Katheterlage ist dadurch ausgeschlos-sen. Sieben Bildfelder mit einer Gesamtlän-ge von knapp 1,20 m können aufgenommen werden. Für jeden Aufnahme-Schritt sind kV-Wert, Bildfrequenz und individuelle Ein-blendung programmierbar. Eine Automatik korrigiert diese Werte während des Untersuchungsablaufs mit der Schrittverschiebung. Ab dem dritten Schritt schwenkt zusätzlich ein Aluminiumkeil in den Strahlengang (zur

Abb. 1. Der Angiographie-Arbeitsplatz CompatixA ist ein Röntgengerät von Siemens für angiographische Untersuchungen in Indirekt-Technik. Der sehr kompakte Aufbau und die Anordnung Obertisch-Strah-lerlUnterirdisch-Bildverstärker ermöglichen guten Zugang zum Patienten.

Neuigkeiten für die klinische Praxis

313

Ausblendung der Direktstrahlung zwischen den Beinen). Indirektaufnahme-Technik mit einer vollautomatischen 100-mm-Blattfilm-Kamera bedeutet ständige Betriebsbereit-schaft und einfache Handhabung. Der Com-patix A kann durch nur eine Person bedient werden und ist als Notfall-Angiographie-platz geeignet. 
Verminderung der Strahlenexposition des Patienten durch Slot Technik Die Anwendung der Slot-Technik (Belich-tung des Röntgenfilms durch schlitzförmig eingeblendeten Röntgenstrahl) wird heute durch leistungsstarke Röntgengeneratoren mit geglätteter Hochspannung und empfind-licher Belichtungsregelung sowie durch Röntgenstrahler mit hoher Wärmekapazität ermöglicht. Siemens hat einen Slot-Arbeits-platz für die RoutineDiagnostik entwickelt. Die Idee, Röntgenfilme mit einem fort-schreitenden schmalen Strahlenfächer an-stelle des vollflächig projizierten Strahlen-bündels zu belichten, ist schon 80 Jahre alt. Die Vorteile dieser Aufnahmetechnik -nämlich besserer Bildkontrast durch ge-ringere Streustrahlung und höhere Bild-schärfe aufgrund der günstigeren geometri-schen Projektionsbedingungen - erkannte man bereits in den Anfangsjahren der medi-zinischen Röntgendiagnostik. Eine breite Einführung dieses Verfahrens scheiterte je-doch an der bislang ungenügenden Belast-barkeit von Röntgenröhre und -generator. Die moderne Röntgentechnologie schuf Ge-neratoren mit hoher Leistung und gutem Wirkungsgrad durch Multipuls-Hochspan-nung. Dadurch konnte die Dosisausbeute der Röntgenröhre erheblich verbessert werden. Röntgenstrahler mit hoher Wärmekapazität (z. B. durch Graphit-Anode) ver-kraften heute auch im Routinebetrieb die Belastung durch SlotAufnahmen. Niedrige Patientendosis ist ein weiterer Vorteil von Slot-Aufnahmen. Das bei vollflächiger Pro-jektion notwendige Streustrahlenraster fällt hier weg; die Strahlenexposition des Patienten ist bis auf die Hälfte reduziert. Für den Röntgen-Aufnahmeplatz Multix CPS hat Siemens Ausbaustufen entwickelt, die an diesem Gerät neben konventioneller Raster- und Schichtaufnahmetechnik auch Slot-Aufnahmen ermöglichen. Aus dem Strahlenbündel der Röntgenröhre wird mit einer (primären) Schlitzblende ein schmaler Strahlenfächer ausgeblendet, der beim Schwenken des Röntgenstrahlers über den Film streicht und ihn fortschreitend belichtet. Eine synchron mitlaufende (sekundäre) Blende direkt über der Filmkassette be-grenzt den Strahl auf $10 \mathrm{~mm}$ Breite und schirmt den Film gegen Streustrahlung ab. Das fortschreitende Belichten des Films durch Schwenken des Röntgenstrahlers hat den weiteren Vorteil, daß der projizierte Brennfleck seine Größe beibehält. Mit an-deren Worten, das gesamte Filmformat wird immer mit der geometrisch günstigeren Brennfleckgröße des Zentralstrahles belich-tet. Das ergibt Bilder mit einer höheren und gleichmäßigeren Zeichenschärfe als bei konventioneller Aufnahmetechnik. Das Anwendungsgebiet der Slot-Technik liegt vor-wiegend im Bereich des Schädels, der Wir-belsäule, der Lunge und der Extremitäten.

Abb. 2. Röntgen-Arbeitsplatz (Siemens), mit dem Röntgenaufnahmen sowohl in konventioneller Technik als auch in Slot-Technik (Belichtung des Röntgenfilms durch schlitzförmig eingeblendeten Röntgenstrahl) ausge-führt werden können. Verantwortlich für die Zusammenstellung: Dr. med. U. Sprandel, München

Vorankündigung

Deutsche Krebsgesellschaft

17. Krebskongreß München vom 7.-10. März 1984, Sheraton-Hotel Nähere Informationen erhalten Sie beim Generalsekretär der Deutschen Krebsgesellschaft e.V. c/o Pathologisches Institut der Medizin. Hochschule Gutschowstraße 8, 3000 Hannover 61 Telefon (0511) 532-4539 